\title{
PROPAGATION AND CANCELLATION OF SINGULARITIES IN A CLASS OF FUCHSIAN OPERATORS AND THEIR PERTURBATIONS
}

\author{
B. R. NAGARAJ AND RAHUL JAIN
}

Received 6 March 2006; Accepted 12 March 2006

We obtain some new necessary conditions for wavefront propagation in the noninvolutive Fuchsian operator class $t \partial^{2} / \partial t^{2}+\lambda\left(x, D_{x}\right) \partial / \partial t+\beta\left(x, D_{x}\right)$. The point of view here is to show that cancellation of propagation of a new singularity in some semilinear systems can be arranged by means of Fuchsian perturbation admitting exact (left and right) microlocal parametrices.

Copyright @ 2006 Hindawi Publishing Corporation. All rights reserved.

\section{Introduction}

This paper is addressed to the study of some new necessary conditions for the $\tau$-axis propagation in the noninvolutive operator class $\widetilde{M}_{\lambda, \beta}=t \partial^{2} / \partial t^{2}+\lambda\left(x, D_{x}\right) \partial / \partial t+\beta\left(x, D_{x}\right)$ treated in the paper of Nagaraj [3]. In particular, the method of constructing analytical solutions to $\widetilde{M}_{\lambda, \beta}=0$ (see [3, expression (5.17)]) is examined so as to obtain new necessary conditions for $\tau$-propagation in the context of [3, Theorem 5.1], notably when the wavefront sets of $\lambda$ and $\beta$ intersect near $\left(x_{0}, \xi_{0}\right)$. This study was motivated while attempting to cancel the propagation of a newly formed singularity in the following second-order degenerate (Fuchsian) semilinear hyperbolic system in the real space-time variables $x, t$ :

$$
\begin{gathered}
\frac{\partial u}{\partial t}-\frac{\partial u}{\partial x}=0 \\
\frac{\partial v}{\partial t}+\frac{\partial v}{\partial x}=0 \\
{\left[(t-1) \frac{\partial^{2}}{\partial t^{2}}+\lambda \frac{\partial}{\partial t}+\beta\right] w=u \cdot v}
\end{gathered}
$$

where $\lambda, \beta$ are arbitrary real or complex constants. As initial values for the solution $u(x, t)$, $v(x, t), w(x, t)$ of the system $(1.1)$, we take $(H=$ Heaviside function $)$

$$
u(x, 0)=H(1+x)
$$

Hindawi Publishing Corporation

International Journal of Mathematics and Mathematical Sciences

Volume 2006, Article ID 65287, Pages 1-9

DOI 10.1155/IJMMS/2006/65287 


$$
\begin{array}{r}
v(x, 0)=H(1-x), \\
w(x, 0)=0=\frac{\partial w}{\partial t}(x, 0) .
\end{array}
$$

We note that for the case $t$ close to 1 (neglecting the second-order term; see Remark 3.2 below), $\lambda=1, \beta=0$ produces a well-known semilinear equation in which the nonlinearity $u \cdot v$ produces a new singularity in $w$ at $(x=0, t=1)$ which is propagated along the half $t$-axis $t \geq 1$; see, for instance, Taylor [4]. Now in a practical model such as simultaneous explosion or seismic activity, one may wish to cancel the propagation along $t \geq 1$ and we demonstrate that this can be done by a second-order Fuchsian perturbation introduced around the singularity $(t=1)$ and matching it with the nonlinearity as in the system (1.1); in principle, a perturbation here should mean addition to $\partial w / \partial t$ by $M_{(0, \beta)} w$ (see (1.3) below). Notice that a first-order Fuchsian perturbation around $(t=1)$ will be degenerate hyperbolic at $(t=0)$ and not at the singularity $(t=1)$.

The cancellation of propagation along the base $t$-axis of the wavefront point at the triple characteristic $\left(x=x_{0}, t=1, \xi=\xi_{0}, \tau=0\right)$ in $\operatorname{WF}(w)$, the wavefront set of the solution in the perturbed system (1.1), is demonstrated by the use of an appropriate exact (both left and right) parametrix for the noninvolutive Fuchsian operator class:

$$
M_{(\lambda, \beta)}=(t-1) \frac{\partial^{2}}{\partial t^{2}}+\lambda \frac{\partial}{\partial t}+\beta
$$

where $\lambda, \beta$ are real or complex constants. We remark that operators of type (1.3) are microlocal equivalents via the MKE (Maslov-Kuranishi-Egorov) setup, of a more general class of operators $R=P Q^{2}+A Q+B$ where $P, Q$ are pseudodifferential operators with real principal symbols of orders 0 and 1 , respectively, and $A, B$ are operators of order 0 , with $P, Q$ having noninvolutive intersection, that is, the Poisson bracket of their principal symbols is nonzero at some point $z_{\circ}$ at which both the principal symbols vanish (see [3]). We emphasise that we are here in a situation where we have to establish propagation or no propagation of a point that is common to the wavefront sets of $w$ and the right-hand side $u \cdot v$ in (1.1); normally the propagation results are stated outside the wavefront set of the right-hand side of the equation.

While attempting to avoid the base propagation in the class (1.3), it is natural to ask for the very general circumstances, allowing $\lambda, \beta$ to be classical pseudodifferential operators of order 0 in the $x$-variables, under which there is a possibility of $\tau$-axis propagation of the wavefront set of the general solution. The reason here is that if the singularity is made to stay put and not propagate in the base, then there is flexibility for it to propagate along the other (fiber) directions.

Addressing these questions, the paper is organised as follows. In Section 2, for the case of $\lambda\left(x, D_{x}\right), \beta\left(x, D_{x}\right)$ being classical pseudodifferential operators of order zero in $x$-variables (dimension $x$ can be $\geq 1$ here), we give new conditions (see [3, Theorems 5.1, and 5.2]) under which $t$-analytical solutions to the homogeneous equation

$$
\widetilde{M}_{(\lambda, \beta)} u=0
$$


can be constructed so that $\tau$-axis propagation is then not possible in general; contrapositively this gives interesting new necessary conditons for $\tau$-axis propagation. In Section 3, we demonstrate by wavefront estimate for an explicit choice of a two-sided parametrix for $M_{(1,0)}$ that the forward propagation along the $t$-axis, $t>1$, does not take place although there is a possibility of $\tau$-axis propagation, as the wavefront estimates indicate. In Section 4, we observe that such a cancellation effect is indeed there, even if we consider perturbation by $M_{(\lambda, \beta)}$, where for the purpose of exactness of microlocal parametrices, $\lambda$, $\beta$ are only taken arbitrary constants, real or complex. It is also appropriate here to note that in a "correct sense" of perturbation, $\lambda$ can be introduced only as a nonzero multiplication on both sides of the equation $\partial w / \partial t=u \cdot v$. Thus if $\lambda=\lambda\left(x, D_{x}\right)$ were to be an operator in the $x$-variables, such an operator action on $u \cdot v$ may in fact smooth out or shift away the wavefront points of $u \cdot v$ unless of course $\lambda$ is chosen to be hypoelliptic near the point in question; notice that there are indeed hypoelliptic unsolvable operators.

\section{Necessary conditions for $\tau$-propagation in $\widetilde{M}_{(\lambda, \beta)}=t \partial^{2} / \partial t^{2}+\lambda\left(x, D_{x}\right) \partial / \partial t+\beta\left(x, D_{x}\right)$}

We recall from [3] that $\tau$-propagation, that is, propagation along the null bicharacteristics of the symbol $t$, is established under the noninteraction condition (near the triple characteristic $\left.z_{0}=\left(x=x_{0}, t=0, \xi=\xi_{0}, \tau=0\right)\right) \operatorname{WF}(\lambda) \cap \mathrm{WF}(\beta)=\phi$ and under the "discrete condition" of $\lambda_{0}$ (principal symbol of $\lambda$ ) avoiding a subset of the integers; see [3, Theorem 5.1] of the paper. Also, again under the noninteraction condition when $\lambda$ is now smoothing and $\beta$ elliptic, $\tau$-propagation is established in [3, Theorem 5.2]. It is our purpose in this section to establish necessary conditions for $\tau$-propagation which include the "interaction condition" when the wavefront sets of $\lambda$ and $\beta$ intersect near $z_{0}$. We begin by looking at sufficient conditions for "no $\tau$-propagation." It will be convenient here to recall certain remarks made at the end of paper, [3, page 573], concerning failure of solvability in the system (5.17) of [3] therein; we are now ready to state the following.

Theorem 2.1. Let $\left(x_{\circ}, \xi_{\circ}\right) \in W F(\lambda) \cap W F(\beta)$, and if (at all) $k-1+\lambda_{\circ}\left(x_{\circ}, \xi_{\circ}\right)=0$ for some integer $k \geq 0$, then suppose $\lambda+k-1$ is solvable near $x_{\circ}$. Then $\widetilde{M}_{\lambda, \beta}$ does not admit $\tau$ propagation.

Proof. We follow the method of proof of [3, Theorem 5.1] and consider with the notation thereof the expression $u(x, t)=u_{\circ}(x)+\sum_{m=1}^{\infty} u_{m}(x) t^{m},\left(x_{0}, \xi_{0}\right) \in \mathrm{WF}\left(\beta u_{0}\right)$, and the set of equations provided by $\widetilde{M}_{\lambda, \beta} u=0$ :

$$
\begin{aligned}
\lambda(x, D) u_{1} & =-\beta(x, D) u_{\circ} \\
m[\lambda(x, D)+m-1] u_{m} & =-\beta(x, D) u_{m-1}, \quad m \geq 1 .
\end{aligned}
$$

Now if all the operators $\lambda(x, D)+m-1$ are elliptic near $\left(x_{\circ}, \xi_{\circ}\right)$, which is in fact a discrete condition on $\lambda_{\circ}$, then from (5.18) in [3], we have

$$
\begin{gathered}
u_{m}=L_{m} u_{\circ} \bmod C^{\infty} \text { near } x_{\circ}, \\
L_{m}=\frac{(-1)^{m}}{m !}(\lambda+m-1)^{-1} \beta(\lambda+m-2)^{-1} \beta \cdots \lambda^{-1} \beta,
\end{gathered}
$$


and the convergence for $u$ is given in [3]. If now $\lambda_{0}\left(x_{0}, \xi_{0}\right)+k-1=0$ for some $k \geq 0$, then clearly, as the discrete conditon is violated at only this $k$ ( $\lambda$ not smoothing near $\left.z_{0}\right)$, we can solve for

$$
\begin{gathered}
u_{m}=L_{m} u_{\circ} \bmod C^{\infty} \quad \text { near } x_{\circ}, 1 \leq m<k \\
u_{k} \text { solves } k[\lambda(x, D)+k-1] u_{k}=-\beta(x, D) u_{k-1} \quad \text { near } x_{\circ}, \\
u_{k+m}=\widetilde{L}_{k+m} u_{k} \bmod C^{\infty} \quad \text { near } x_{\circ}, m \geq 1, \\
\widetilde{L}_{k+m}=\frac{(-1)^{m}}{m !}(\lambda+k+m-1)^{-1} \beta \cdots(\lambda+k+1)^{-1} \beta(\lambda+k)^{-1} \beta .
\end{gathered}
$$

It is therefore clear from $(2.3)$ that the series for $u(x, t)$ has $L^{2}$ convergence on compact $t$ sets. It follows that $\left(x_{\circ}, 0, \xi_{\circ}, 0\right) \in \mathrm{WF}(u)$ as reasoned in the proof of [3, Theorem (5.1)] which is not $\tau$-propagated, completing the proof.

Remark 2.2. With regard to the statement of Theorem 2.1, we note that if $\lambda$ verifies the discrete condition instead, then the fact that $\beta$ is not smoothing near $\left(x_{0}, \xi_{0}\right)$ implies the assertion in view of [3, Theorem 5.1]. We also note from Theorem 2.1 that under the "interaction condition" $\operatorname{WF}(\lambda) \cap \mathrm{WF}(\beta) \neq \phi$, if $\widetilde{M}_{(\lambda, \beta)}$ admits $\tau$-propagation, then this necessarily implies that $\lambda+k-1$ is unsolvable for any integer $k \geq 0$ (at which $\left.\lambda_{\circ}\left(x_{\circ}, \xi_{\circ}\right)+k-1=0\right)$ near the "interacting point" $x_{\circ}$ (i.e., $\left.\left(x_{\circ}, \xi_{\circ}\right) \in \mathrm{WF}(\lambda) \cap \mathrm{WF}(\beta)\right)$. We may thus take note of the "weaker" necessary conditions for $\tau$-propagation given in the [3, Theorems 5.1 and 5.2].

\section{Perturbation by $M_{(0,0)}$}

We first note that the "Hörmander product" $u \cdot v$ in (1.1) is well defined at the level of wavefront sets, since, solving for $u$ and $v$ in (1.1) respectively as $H(1-t \mp x)$ in terms of the Heaviside function $H$ it is easy to see

$$
\begin{aligned}
& \mathrm{WF}(u) \subset\{(1-t, t, \tau \neq 0, \tau)\}, \\
& \mathrm{WF}(v) \subset\left\{\left(t-1, t, \tau_{1} \neq 0,-\tau_{1}\right)\right\} .
\end{aligned}
$$

Thus clearly the fiber sum $\left(\tau+\tau_{1}, \tau-\tau_{1}\right) \neq(0,0)$, so that $u \cdot v$ is well defined. Now one solves for $w$ from the equation $\partial w / \partial t=u \cdot v$ by simple integration noting that the integrand is smooth for $t<1$ in $(x, t)$ space, outside the union of the lines $1-t \pm x=0$. The "joint" singularity in $u$ and $v$ at $x=0, t=1$ is picked up by $w$ (in view of microlocal regularity) and propagated along the half $t$-axis, $t \geq 1$, as per the solution $w(x, t)=1-$ $|x|,|x| \leq 1$; see [4]. A question arises as to why the singularity in $w$ is not propagated in both directions of the $t$-axis from $t=1$ in view of Hörmander's propagation theorem; but this cannot be since propagation results are normally valid outside the wavefront set of the right-hand side, which is not the case here. Now we allow the second-order Fuchsian 
perturbation near the singularity $t=1$ and study the equation

$$
\begin{gathered}
M_{(1,0)} w=u \cdot v \\
w=\frac{\partial w}{\partial t}=0 \quad \text { on } t=0 .
\end{gathered}
$$

Solving (3.2) "factor-wise," we easily see that

$$
w(x, t)=\int_{0}^{t}\left(\frac{1}{r-1} \int_{0}^{r} u(s) \cdot v(s) d s\right) d r .
$$

Now by smoothness of the integrand in $t<1$ as observed in the paragraph following (3.1), it follows that $w$ is smooth in the interior of the triangular region bounded by the $x$-axis and the lines $1-t \pm x=0$ passing through $(t=1, x=0)$. In particular, the singularity in an "extended" solution near $t=1$ is not per se by uniqueness of solution of (3.2) propagated in $t<1$; this is also confirmed by microlocal analysis below (see the paragraph above Theorem 3.1). A method of extension of this solution to a neighbourhood of $t=1$ is given in (3.5) below, which is well defined in terms of the kernels (3.6). Now as to whether this singularity in $w$ at $t=1$ propagates in $t>1$ along the $t$-axis, we show that such is not the case. It will now be our purpose to construct appropriate two-sided microlocal parametrices for $M_{(1,0)}$ in such a way that as far as the "base" $t$-propagation is concerned, the singularity in $w$ at $t=1, x=0$ stays put there. We note that both the necessary conditions of Theorem 2.1 are satisfied for the operator $M_{(1,0)}$, giving "hint" of flexibility for $\tau$-propagation.

The translation $t \rightarrow t-1$ gives

$$
\widetilde{M}_{(1,0)} \widetilde{w}=\left[t \frac{\partial^{2}}{\partial t^{2}}+\frac{\partial}{\partial t}\right] \widetilde{w}=(\widetilde{u v})=f(x, t)
$$

where $(\widetilde{u v})$ has now a "base" singularity at $t=0, x=0$. Now a formal solution to (3.4) can be written:

$$
\tilde{w}(x, t)=\int^{t}[\log |t|-\log |s|] f(x, s) d s
$$

This formal solution leads to well-defined distribution kernels which represent the following microlocal parametrices, each being both a left as well as a right exact inverse for $\widetilde{M}_{(1,0)}$ :

$$
\left[\log |t| \otimes 1_{s}-1_{t} \otimes \log |s|\right] \cdot H(t \pm s) \otimes \delta(x-y) .
$$

The fact that the kernels (3.6) are left parametrices as well follows from their action in the $s$-variable on $\widetilde{M}_{(1,0)} f(x, s)$ and integrating by parts. It can be verified that the $(t, s)$ parts of the distributions in (3.6) are well defined in terms of the Hörmander product condition, since, for instance, $\operatorname{WF}(H(t \pm s)) \subset\{(t, \mp t,-\tau, \tau): t \in \mathbb{R}, \tau \neq 0\}$.

Now consider the kernel

$$
K_{(1,0)}=\left[\log |t| \otimes 1_{s}-1_{t} \otimes \log |s|\right] \cdot H(t-s) \otimes \delta(x-y) .
$$


Using the fact that

$$
\mathrm{WF}(\log |t|) \subset\left\{(0, \tau): \tau \in \mathbb{R}^{*} \backslash 0\right\}
$$

and recalling the "tensor and Hörmander product formulae" for wavefront sets, we obtain the following wavefront estimate on the kernel $K_{(1,0)}$ (in the following $\xi \neq 0$ when it occurs below):

$$
\begin{aligned}
\mathrm{WF}\left(K_{(1,0)}\right) & \subset\left\{(x, 0, \xi, \tau ; x, s,-\xi, 0): s \in \mathbb{R}, \tau \in \mathbb{R}^{*} \backslash 0\right\} \\
& \cup\left\{(x, t, \xi, 0 ; x, 0,-\xi, \sigma): t \in \mathbb{R}, \sigma \in \mathbb{R}^{*} \backslash 0\right\} \\
& \cup\left\{(x, t, \xi, \tau ; x, t,-\xi,-\tau):(t, \tau) \in T^{*}(\mathbb{R}) \backslash 0\right\} \\
& \cup\left\{(x, 0, \xi, \tau ; x, 0,-\xi, \sigma): \tau \in \mathbb{R}^{*}, \sigma \in \mathbb{R}^{*} \backslash 0\right\} \\
& \cup\left\{(x, 0, \xi, \tau ; x, 0,-\xi, \sigma): \tau, \sigma \in \mathbb{R}^{*}, \tau \neq 0\right\} \\
& \cup \text { of sets above in each of which } \xi=0 \\
& \cup\{(x, t, \xi, 0 ; x, s,-\xi, 0): t \geq s\} .
\end{aligned}
$$

Now from the equation

$$
\widetilde{w}(x, t)=K_{(1,0)} \widetilde{M}_{(1,0)} \widetilde{w}=K_{(1,0)}(\widetilde{u v})
$$

we obtain, applying wavefront calculus (composition formula),

$$
\mathrm{WF}(\widetilde{w})=\left[\mathrm{WF}^{\prime}\left(K_{(1,0)}\right) \circ \mathrm{WF}(\widetilde{u v})\right] \cup\left[\mathrm{WF}_{(x, t)}^{\prime}\left(K_{(1,0)}\right)\right]
$$

in relation to the singular point $\left(0,0, \xi_{0}, 0\right)$ of $(\widetilde{u v})$, the following: in order that $\left(0,0, \xi_{0}, 0\right)$ be a possible first coordinate in the union (3.9), which must be the case since $\widetilde{w}$ has such a singular point, it is necessary that the sets $\left\{\left(0, s,-\xi_{0}, 0\right): 0 \geq s\right\}$ and $\left\{\left(0,0,-\xi_{0}, \sigma\right): \sigma \neq 0\right\}$ form second coordinates with $\left(0,0, \xi_{0}, 0\right)$ in the union (3.9). But, by the "mapping principle" of the composition, since $(\widetilde{u v})$ does not have singularities on the $t$-axis other than at $t=0$, only one point, that is, $\left(0,0, \xi_{0}, 0\right)$, is relevant in the last set (only this accounts for the "base" propagation) in the union (3.9). Note also that the second set in the union of $\mathrm{WF}(\widetilde{w})$ adds nothing since zero section does not occur in the second 4-tuple in (3.9). We thus see that $\widetilde{w}$ does not have singularities in $t<0$ (a fact already observed in the paragraph following (3.3)) and the same analysis performed using the other parametrix in (3.6) shows that $\widetilde{w}$ has no singularities in $t>0$ as well; we note here that the formula (3.3) works as long as $t \neq 1$ and the distribution kernels constructed in a neighbourhood of $t=0$ are exact inverses and thus only one solution is possible, either in $t<0$ or in $t>0$. Thus the singularity in $\widetilde{w}$ at the point $\left(0,0, \xi_{0}, 0\right)$ can at best propagate only along the $\tau$ axis and we have achieved the cancellation effect in fact in $w$ along the $t$-axis. We thus obtain the following. 
Theorem 3.1. For the degenerate hyperbolic semilinear system on $\mathbb{R}_{x} \times \mathbb{R}_{t}$,

$$
\begin{gathered}
u_{t}-u_{x}=0, \\
v_{t}+v_{x}=0, \\
(t-1) \partial_{t}^{2} w+\partial_{t} w=u v, \\
u(x, 0)=H(1-x), \\
v(x, 0)=H(1+x), \\
w(x, 0)=\frac{\partial w}{\partial t}(x, 0)=0,
\end{gathered}
$$

the joint singularity at $x=0, t=1$ is not propagated along the $t$-axis for $t>1$, that is $(x, t)$ projection of

$$
\mathrm{WF}\left(\begin{array}{c}
u \\
v \\
w
\end{array}\right)
$$

intersects the $t$-axis only at $x=0, t=1$. (Thus there is "guided" propagation only along the characteristics $t-1 \pm x=0$. Such is not the case if the term $(t-1) \partial_{t}^{2} w$ is absent, in which case new singularity at $x=0, t=1$ occurs and will be propagated along the $t$-axis for $t \geq 1$.)

Remark 3.2. From the expression (3.5) we may formally compute at $x=0$,

$$
\begin{gathered}
w_{t}=\frac{1}{t} \int^{t} f(s) d s, \\
t w_{t t}=-\frac{1}{t} \int^{t} f(s) d s+f(t) \longrightarrow 0 \quad \text { as } t \longrightarrow 0,
\end{gathered}
$$

by L'Hospital's rule. Thus for our choice of $f=H(-t+x) H(-t-x), t w_{t t}(0, t)$ is small for small $t$; hence the term "perturbation" makes sense.

\section{Perturbation by $M_{(\lambda, \beta)}$}

Given the equation $\partial w / \partial t=u \cdot v$, one can obviously multiply both sides by an arbitrary nonzero constant $\lambda$, real or complex, without affecting the singular nature of the solution $w$, in relation to formation and propagation of new singularities due to nonlinear interaction in $\lambda \cdot u \cdot v$. This suggests that we consider the perturbed system as in (1.1), where $\lambda, \beta$ are taken as arbitrary real or complex constants, to examine whether the cancellation effect is still there. We demonstrate that this is so by the use of the Bessel integral method of eliciting the singularities, as is done in [3, Section 3]. Accordingly, the solution of $\widetilde{M}_{(\lambda, \beta)} \widetilde{w}=(\widetilde{u v})=f(x, t)$ may be written in the form (see [3, expression (3.3)])

$$
u(x, t)=\frac{2}{c} t^{k / 2} \int^{t}\left[J_{k}(2 \sqrt{\beta s}) N_{k}(2 \sqrt{\beta t})-J_{k}(2 \sqrt{\beta t}) N_{k}(2 \sqrt{\beta s})\right] s^{-k / 2} f(x, s) d s .
$$


Now, introducing limits from the "upper and lower" half spaces in the expression (4.1) (see $[1,2]$ and $[3$, expressions $(3.7),(3.8)]$ ), we obtain the following four right which are also left parametrices:

$$
\begin{gathered}
H(t \pm s) \cdot F_{(\lambda, \beta)}^{ \pm}(t, s) \otimes \delta(x-y) \\
F_{(\lambda, \beta)}^{ \pm}(t, s)=-\frac{2}{c} g(2 \sqrt{\beta t})(t \pm i 0)^{1-\lambda} \otimes \tilde{g}(2 \sqrt{\beta s})(s \pm i 0)^{\lambda-1}
\end{gathered}
$$

(mod a smooth function).

It is noticed that the distribution kernels in (4.2) are well defined since the Hörmander product condition is verified.

Now, following $[1,2]$, it is observed that the wavefront estimates on these kernels indicate that at most the union of two transverse half characteristics through the triple characteristic $\left(0,0, \xi_{0}, 0\right)$ is involved for each parametrix; we thus observe on the lines of proof of Theorem 3.1 that there is no propagation along the base $t$-axis and this leads to the following.

Theorem 4.1. For the degenerate hyperbolic semilinear system on $\mathbb{R}_{x} \times \mathbb{R}_{t}$ with $\lambda$, $\beta$ arbitrary constants, real or complex,

$$
\begin{gathered}
u_{t}-u_{x}=0, \\
v_{t}+v_{x}=0, \\
(t-1) \partial_{t}^{2} w+\lambda \partial_{t} w+\beta w=u \cdot v, \\
u(x, 0)=H(1-x), \\
v(x, 0)=H(1+x), \\
w(x, 0)=\frac{\partial w}{\partial t}(x, 0)=0,
\end{gathered}
$$

the joint singularity at $x=0, t=1$ is not propagated along the $t$-axis for $t>1$, that is, $(x, t)$ projection of

$$
\mathrm{WF}\left(\begin{array}{c}
u \\
v \\
w
\end{array}\right)
$$

intersects the $t$-axis only at $x=0, t=1$. (Thus there is "guided" propagation only along the characteristics $t-1 \pm x=0$. Such is not the case if the term $(t-1) \partial_{t}^{2} w$ is absent, in which case $(\lambda \neq 0)$ new singularity at $x=0, t=1$ occurs and will be propagated along the $t$-axis for $t \geq 1$.)

Remark 4.2. Our procedure of perturbation in this section has centred around "multiplication" by $\lambda$ of the equation $\partial w / \partial t=u \cdot v$. Obviously, unless by elliptic (or indeed hypoelliptic) multiplication, we cannot use action by an arbitrary pseudodifferential operator $\lambda$ 
in the $x$-variables, in order to maintain wavefront sets. With such perturbations however, microlocal analysis in [3] indicates that we do indeed have validity of Theorem 4.1.

\section{Acknowledgments}

We thank Professors Mythily Ramaswamy and Veerappa Gowda for their constructive criticism, interest, and encouragement. We thank Professor S. Kichenassamy (University of Reims) for giving one of the authors (Rahul Jain) an opportunity to present this work in a seminar at Reims (France) and for his useful suggestions.

\section{References}

[1] N. Hanges, Parametrices and propagation of singularities for operators with noninvolutive characteristics, Indiana University Mathematics Journal 28 (1979), no. 1, 87-97.

[2] _ Propagation of singularities for a class of operators with double characteristics, Seminar on Singularities of Solutions of Linear Partial Differential Equations (Inst. Adv. Study, Princeton, NJ, 1977/1978), Ann. of Math. Stud., vol. 91, Princeton University Press, New Jersey, 1979, pp. 113-126.

[3] B. R. Nagaraj, Propagation of singularities for a class of Fuchsian operators, Communications in Partial Differential Equations 23 (1998), no. 3-4, 547-575.

[4] M. E. Taylor, Pseudodifferential Operators and Nonlinear PDE, Progress in Mathematics, vol. 100, Birkhäuser Boston, Massachusetts, 1991.

B. R. Nagaraj: TIFR Centre, Indian Institute of Science, 560012 Bangalore, India E-mail address: brn@math.tifrbng.res.in

Rahul Jain: TIFR Centre, Indian Institute of Science, 560012 Bangalore, India

E-mail address: rahul@math.tifrbng.res.in 


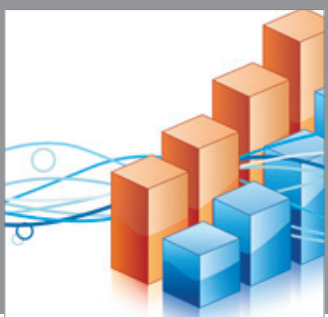

Advances in

Operations Research

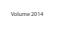

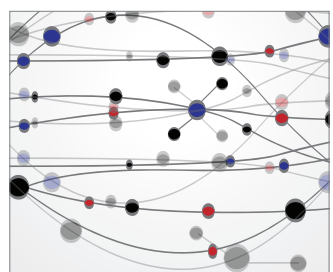

\section{The Scientific} World Journal
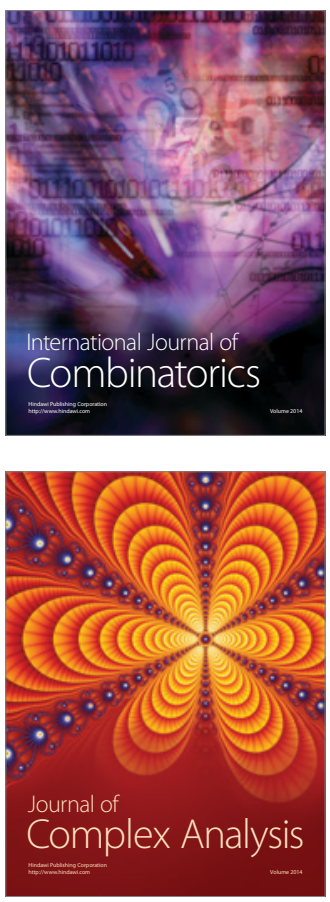

International Journal of

Mathematics and

Mathematical

Sciences
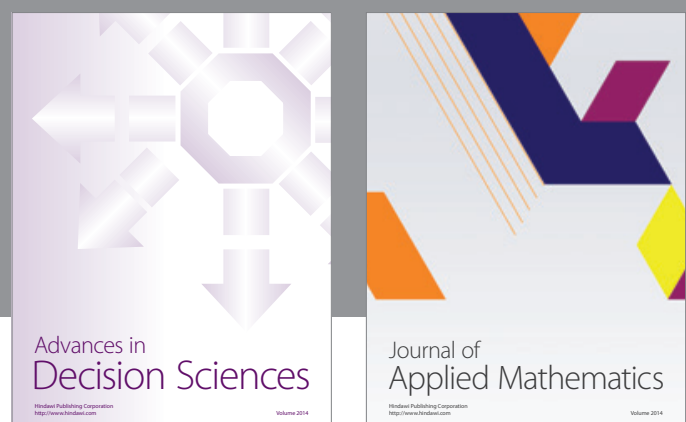

Journal of

Applied Mathematics
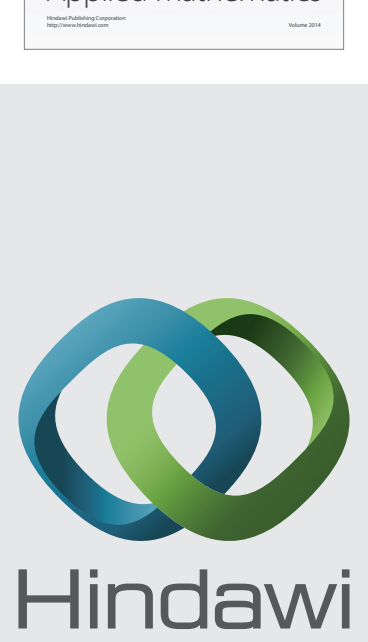

Submit your manuscripts at http://www.hindawi.com
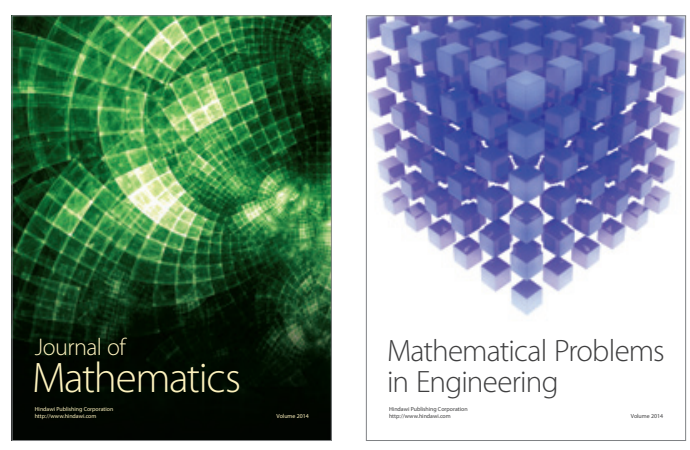

Mathematical Problems in Engineering
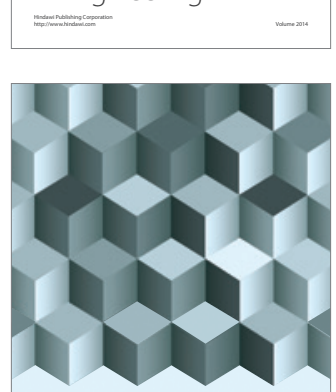

Journal of

Function Spaces
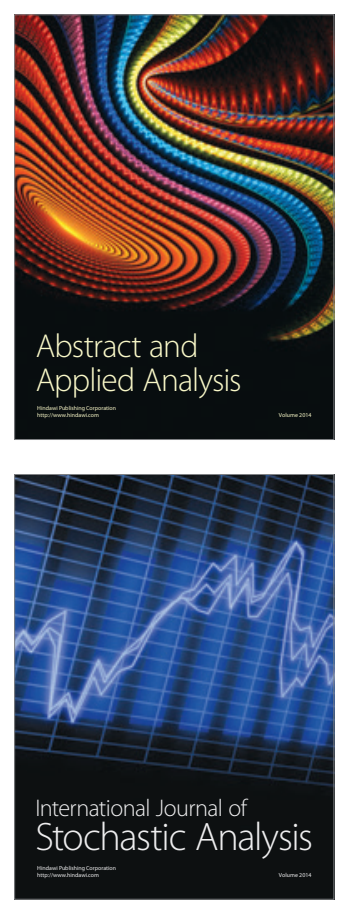

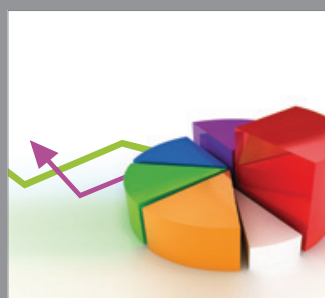

ournal of

Probability and Statistics

Promensencen
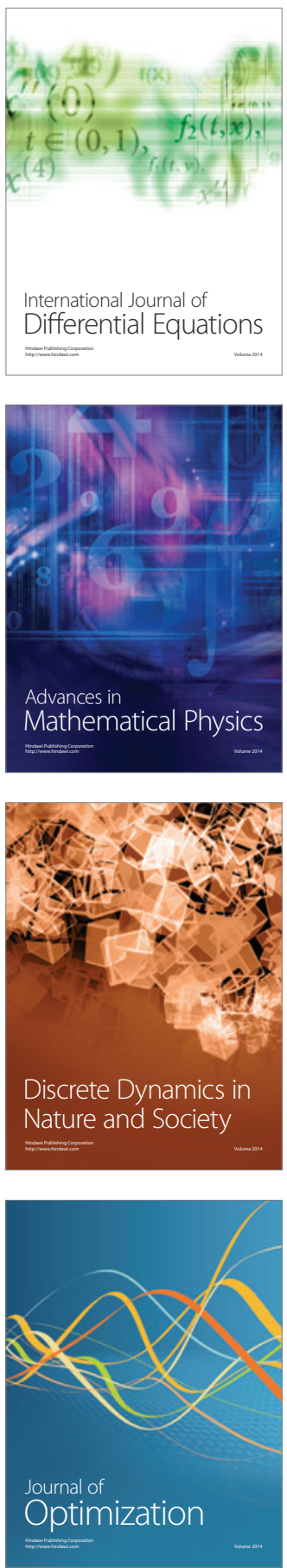